\title{
Rehabilitación de una presa de residuos mineros mediante la aplicación de lodo de mármol y purín de cerdo para el desarrollo de una fitoestabilización asistida
}

\author{
R. Zornoza(1), Á. Faz'(1), S. Martínez-Martínez(1), J.A. Acosta(1), M.D. Gómez-López ${ }^{(1)}$, M.Á. Muñoz ${ }^{(1)}$, \\ R. Sánchez-Medrano(2), F. J. Murcia ${ }^{(3)}$, F.J. Fernández Cortés ${ }^{(4)}$, E. López Martínez ${ }^{(4)}$, A. Espín de Gea ${ }^{(4)}$
}

(1) Gestión, Aprovechamiento y Recuperación de Suelos y Aguas.

Dpto. de Ciencia y Tecnología Agraria. Universidad Politécnica de Cartagena. Paseo Alfonso XIII, 48, 30203 Cartagena. España. raul.zornoza@upct.es

(2) Servicio de Minas.

Dirección General de Industria, Energía y Minas de la Consejería de Industria, Turismo, Empresa e Innovación de la Región de Murcia. C/ Nuevas tecnologías s/n, 30005 Murcia. España

(3) Dirección General de Medio Ambiente de la Consejería de Agricultura y Agua de la Región de Murcia.

C/ Catedrático Eugenio Úbeda 3, 30071 Murcia. España

(4) Asociación Empresarial de Investigación Centro Tecnológico del Mármol, Piedra Natural y Materiales. Ctra. de Murcia s/n, 30430 Cehegín, Murcia. España

\section{RESUMEN}

Las acumulaciones de residuos mineros abandonados presentan riesgos ambientales y de salud pública por la potencial transferencia de metales pesados tóxicos a través de la erosión hídrica y eólica o lixiviación. Para reducir estos riesgos, se ha desarrollado una estrategia de rehabilitación en una presa de residuos mineros basada en la fitoestabilización asistida. A lo largo de 2011 se aplicó sobre la superficie de la presa lodo de mármol $\left(6.7 \mathrm{~kg} \mathrm{~m}^{-2}\right)$, purín de cerdo $\left(1.7,7 \mathrm{~L} \mathrm{~m}^{-2}\right.$ en julio y $2.6 \mathrm{~L} \mathrm{~m}^{-2}$ en septiembre), y la fase sólida de purín de cerdo $\left(7 \mathrm{~kg} \mathrm{~m}^{-2}\right)$. En primavera de 2012 se introdujeron trece especies vegetales autóctonas. Durante dos años (20122013) se ha monitorizado con carácter semestral la evolución de diferentes propiedades edáficas y la fracción biodisponible de los metales $\mathrm{Cd}, \mathrm{Pb}$ y $\mathrm{Zn}$. Los resultados mostraron un incremento en el pH (de $\sim 3$ a 7), la estabilidad de agregados (de 7\% a 10-15\%), el carbono orgánico (de $3.5 \mathrm{~g} \mathrm{~kg}^{-1}$ a $3.5 \mathrm{~g} \mathrm{~kg}^{-1}$ ), el nitrógeno total (de $\sim 0.3 \mathrm{~g} \mathrm{~kg}^{-1}$ a $\sim 0.8 \mathrm{~g} \mathrm{~kg}^{-1}$ ) y la capacidad de intercambio catiónico (de $6 \mathrm{cmol}_{+} \mathrm{kg}^{-1}$ a $11 \mathrm{cmol}_{+} \mathrm{kg}^{-1}$ ) con la aplicación de las enmiendas y el desarrollo de la vegetación, mientras que la fracción biodisponible de los metales descendió drásticamente (90-99\%). Por tanto, la estrategia seguida ha resultado positiva para reducir la biodisponibilidad de metales pesados, mejorando la calidad y fertilidad del suelo. Estos resultados son prometedores en áreas donde se generen residuos que puedan ser utilizados para la rehabilitación de suelos afectados por metales pesados, revalorizando los mismos y al convertirse en subproductos de utilidad.

Palabras clave: Presa de residuos, enmiendas, geoquímica, metales pesados, minería.

\section{Reclamation of a mine tailings pond by addition of marble waste and pig slurry for the development of aided phytostabilization}

\section{ABSTRACT}

Abandoned tailings ponds produce environmental and human health hazards due to the transfer of heavy metals through wind and water erosion or leaching. To reduce these hazards, a reclamation strategy has been developed on a tailings pond based on aided phytostabilization. In 2011 marble mud and pig slurry were applied to the surface of a tailings pond and in the spring of 2012 thirteen native vegetal species were introduced. The evolution of different soil properties and the bio-available fraction of the heavy metals $\mathrm{Cd}, \mathrm{Pb}$ and $\mathrm{Zn}$ were monitored for two years (2012-2013). The results showed that the $\mathrm{pH}$, aggregate stability, organic carbon, total nitrogen and cation exchange capacity increased after the application of the amendments and the growth of vegetation, whilst the bio-available fraction of the heavy metals drastically decreased (90-99\%). 
Thus, the strategy followed proved to be positive for reducing the availability of heavy metals and improving soil quality and fertility. These results are promising in areas with extractive activity of carbonated materials, since the generated waste can be used for the reclamation of soils affected by heavy metals, transforming a residual material into a useful by-product.

Keywords: amendments, geochemistry, heavy metals, mining, tailings pond.

\section{ABRIDGED ENGLISH VERSION}

\section{Introduction}

Mine tailings ponds contain materials rich in Fe-oxyhydroxides, sulphides, sulphates, and heavy metals. As a consequence, these soils remain bare and have low soil organic matter content (Conesa et al., 2006). High incidences of wind and water erosion events negatively affect soil, water, vegetation, fauna, and human populations in the surrounding areas (Zanuzzi et al., 2009). The introduction of vegetation cover is needed to reinforce the topsoil, thus reducing soil loss by erosion and reducing runoff velocity. However, owing to metal toxicity and extremely low organic matter content and nutrients, the establishment of vegetation is compromised (Zornoza et al., 2011). Phytostabilization is a green technology that uses selected plant species for in situ heavy-metal immobilization and soil stabilization. The reclamation of abandoned mine sites relies on achieving optimum conditions for plant growth by improving the physical, chemical and biological characteristics of the soil with the use different amendments (Bradshaw and Johnson, 1992). Organic waste such as sewage sludge and animal manure can be used as nutrient sources which stimulate the formation of aggregates (Zanuzzi et al., 2009). Alkaline materials, such as marble waste, are commonly used as amendments for ameliorating the acidic conditions of acid-generating mine waste. The correction of acidity not only enables a wide range of plants to become established but also mitigates metal toxicity and increases plant nutrient availability (Barker, 1997). The objective of this study was to evaluate the effectiveness of a phytostabilization technique combined by the addition of marble waste and pig slurry, for the reclamation of an abandoned mine tailings pond. Furthermore, this rehabilitation strategy increases soil quality and $\mathrm{pH}$, and reduces the availability of heavy metals.

\section{Study area}

The selected tailings pond is located in the Cartagena-La Unión Mining District (SE Spain), where great mining activities have been carried out for more than 2,500 years, up to 1991 when all activities ceased (Fig. 1). The climate for this area is defined as semi-arid Mediterranean, showing a mean annual temperature of $18^{\circ} \mathrm{C}$ and a mean annual precipitation of $275 \mathrm{~mm}$.

\section{Characteristic of the tailings pond}

The material has a sandy loam texture. The residues were mechanically, physically, chemically and biologically deficient, characterized by instability and limited cohesion, with low contents of nutrients and organic matter and high levels of heavy metals. The range of concentrations of total heavy metals was 0.73-6.65 mg $\mathrm{kg}^{-1}$ for Cd; 1246-2048 $\mathrm{mg} \mathrm{kg}^{-1}$ for $\mathrm{Pb}$ and 829-5899 $\mathrm{mg} \mathrm{kg}^{-1}$ for $\mathrm{Zn}$.

\section{Methods}

We used two different amendments (pig slurry and marble (mud) waste $\left(\mathrm{CaCO}_{3}\right)$ ) for reclamation purposes (Table 1). The first application of pig slurry was carried out in July 2011 and the subsequent applications in September and November 2011. We applied the organic amendment in three different instalments to favour a suitable assimilation and stabilization of organic matter and nutrients in the soil before the introduction of vegetation. The marble waste application was carried out in July 2011. After the application of the amendments, all the materials were mixed to a depth of $0-50 \mathrm{~cm}$ to incorporate the amendments into the soil. Marble (mud) was applied at a rate of $6.7 \mathrm{~kg} \mathrm{~m}^{-2}$. This rate was calculated to establish the quantity of lime required to neutralise all the potential acid according to the percentage of sulphides present in the mine soil. We applied $1.7 \mathrm{~L} \mathrm{~m}^{-2}$ of raw pig slurry in July; $2.6 \mathrm{~L} \mathrm{~m}^{-2}$ of raw pig slurry in September and $7 \mathrm{~kg} \mathrm{~m}^{-2}$ of solid phase of pig slurry in November 2011. Between the months of March and April 2012, thirteen native plant species were 
planted or seeded. To monitor the evolution of soil quality, four plots (10 m x $10 \mathrm{~m})$ were established. We carried out an initial soil sampling in October 2010, and four samplings in January and July 2012 and January and July 2013. In each sampling we collected three soil samples per plot, at two different depths (0-15 and 15$30 \mathrm{~cm}$ ). Samples were air-dried and sieved $<2 \mathrm{~mm}$. We measured $\mathrm{pH}$ and electrical conductivity (CE) in deionised water (1:2.5 and 1:5 W/v, respectively), aggregates stability (EA) according to USDA (1999), organic carbon (Corg), inorganic carbon and total nitrogen (Nt) in an elemental CNHS-O analyser (EA-1108, Carlo Elba), the cation exchange capacity (CIC) according to Chapman (1965), the total content of Cd, Pb and $\mathrm{Zn}$ was determined according to Risser and Baker (1990) and the bio-available fraction was extracted with $0.01 \mathrm{M} \mathrm{CaCl}_{2}$ (Pueyo et al., 2004). Heavy metals were measured by atomic absorption spectroscopy (Agilent 7500 CE).

\section{Results and Discussion}

The application of marble waste and pig slurry on the tailings pond has caused changes in all the properties, which have maintained more or less stable during the two years of this study. The $\mathrm{pH}$ significantly increased from 2.89-3.12 before the application of amendments to values close to neutrality after the application (Fig. 4a). This $\mathrm{pH}$ increase is due to the presence of carbonates in soils, which react with acidity returning the soil close to neutrality. The positive significant correlation between $\mathrm{pH}$ and carbonate content supports this assertion. Calcium carbonate content was higher in surface samples, indicating an incorrect homogenization of amendments with depth. On the surface, calcium carbonate increased from 0 up to $5.10 \%$, whilst in the subsurface this increment reached a maximum of $3.43 \%$ (Fig. 4b). These levels have contributed to neutralize acidity at both depths, remaining stable with time. Corg was around $1.5 \mathrm{~g} \mathrm{~kg}^{-1}$ before the application of the amendments, and significantly increased to mean values higher than $3.5 \mathrm{~g} \mathrm{~kg}^{-1}$ (Fig. $4 \mathrm{c}$ ) as a consequence of the application of the pig slurry. Corg has maintained stable with time, even though the normal trend under semi-arid conditions is its mineralization (Zornoza et al., 2012). This is probably due to the development of a vegetation cover which maintains Corg levels by root exudates and litter accumulation (Bouwman and Vangronsveld, 2004) (and the increase of microbial biomass and activity). In fact, the vegetation cover at the end of the study was $65 \%$ of the total surface. The EA has increased in both depths after the application of the amendments (Fig. 4d), with values stable throughout the two years of the study with mean values of between $10-14 \%$. This is due to the incorporation of organic matter and calcium carbonates which contribute to the formation of new aggregates. The CE decreased with the application of amendments (Fig. 5a), due to the application of the marble waste, since there is a negative significant correlation between $\mathrm{CE}$ and $\mathrm{CaCO}_{3}$ content. This may be due to the reaction of $\mathrm{Ca}^{2+}$ with sulphates forming mineral precipitates, or by precipitation of ions by increments in soil pH (Brallier et al., 1996; Fernández-Caliani and Barba-Brioso, 2010). The content of Nt also increased after the incorporation of the amendments (Fig. 5b), with this increment being higher in surface samples. Thus, soil fertility has been enhanced, favouring the development of vegetation. CIC significantly increased with the amendments (Fig. 5c), with no significant differences amongst the different samplings. The application of the pig slurry may have caused this increment which provides functional groups with variable negative charges. The bio-available fraction of heavy metals significantly decreased with the amendments and remained stable during the last year of the study. Reduction of up to $90 \%, 97$ and $99 \%$ of the available fraction of $\mathrm{Cd}, \mathrm{Pb}$ and $\mathrm{Zn}$ was observed, respectively, compared to the initial values (Fig. 6). The decrease in the bio-available fraction of the heavy metals is mainly due to a direct effect of the $\mathrm{pH}$, which favours the processes of adsorption, precipitation and co-precipitation with oxihydroxides; the formation of chelates; the formation of metallic carbonates and, the formation of metallic phosphates (Alvarenga et al., 2008; Liu et al., 2009; Fernández-Caliani and Barba-Brioso, 2010; Zornoza et al., 2013). This is promising in areas with quarries which produce carbonate material (limestone, dolomite, marble) and where there is extra production of organic residues such as pig slurries, since these residues can be used as amendments to reclaim bare main soils with low or no cost (depending on transportation and application), turning them into valuable by-products. The valorization of residues is essential for sustainable development, since land-filling must be reduced to a minimum to prevent an undesirable environmental impact.

\section{Introducción}

En la Región de Murcia (SE España), la intensa actividad minera que tuvo lugar durante el pasado siglo generó grandes cantidades de residuos acumulados en escombreras o balsas. Aunque la actividad minera fue abandonada en 1991, las acumulaciones de residuos mineros aún permanecen en el área, la mayoría de ellos sin rehabilitar. Estas acumulaciones contienen materiales ricos en óxidos e hidróxidos de hierro, sulfuros, sulfatos, metales pesados y metaloides. Como consecuencia, permanecen desprovistos de 
vegetación, con alta acidez debido a la oxidación de los sulfuros y la intensa evaporación. En algunos pantanos se usó agua de mar para el tratamiento del minera. El contenido en nutrientes es bajo (Conesa et al., 2006). Los principales riesgos derivan de las altas tasas de erosión hídrica y eólica, que afectan negativamente a suelos, agua, vegetación, fauna y asentamientos humanos cercanos, por transporte de material con altas concentraciones de contaminantes (Zanuzzi et al., 2009). Como consecuencia, para crear suelo y reducir la erosión es necesario crear una cubierta vegetal estable. Sin embargo, debido a la alta toxicidad de los metales y el extremado bajo contenido en materia orgánica y nutrientes, el establecimiento de la vegetación ha resultado muy limitado por colonización natural o implantación de una cubierta vegetal inadecuada (Zornoza et al., 2011). Las técnicas convencionales de rehabilitación de materiales contaminados por metales pesados incluyen normalmente la eliminación de ese material y su recubrimiento con materiales limpios (Brown et al., 2005), aunque no se consideran la solución disponible más adecuada desde el punto de vista económico y ambiental (Alvarenga et al., 2008). La fitoestabilización asistida es una tecnología emergente de base biológica que usa especies vegetales para la rehabilitación de suelos mediante la estabilización de metales pesados, siendo capaz de inmovilizar metales mediante la adsorción en la rizosfera y acumulación en las raíces, y el uso de enmiendas que mejoren las características físicas, químicas y biológicas del suelo para que las plantas se puedan desarrollar (Bradshaw y Johnson, 1992).

Los residuos orgánicos como el compost, lodo de depuradora o estiércoles ganaderos pueden usarse como fuentes de nutrientes que además estimulan la formación de agregados, el desarrollo de poblaciones microbianas y reducen la disponibilidad de ciertos metales por complejación (Ye et al., 2002; Zanuzzi et al., 2009). Concretamente, la gestión de residuos orgánicos procedentes de la industria porcina representa un serio problema ambiental debido a su gran volumen y poca recuperación. La Región de Murcia presenta más del $8 \%$ de la producción de cerdo en España, con cerca de 2000000 de cabezas. Esta cantidad de animales genera una producción anual de residuos de $6.5 \mathrm{Hm}^{3}$, con problemas de gestión para los productores. El uso de purines porcinos como enmienda orgánica puede solucionar dos problemas: valorización de un subproducto y rehabilitación de zonas mineras degradadas. Tanto el purín bruto como su fase sólida tras una separación física pueden usarse para rehabilitar estas áreas, siempre y cuando se apliquen en dosis adecuadas para evitar un exceso de salinidad y nitratos, altamente móviles con el agua.

La trasformación de los metales en formas menos móviles y por tanto, menos biodisponibles, es una solución posible para la rehabilitación de áreas afectadas por la minería. Los materiales alcalinos, como el residuo del mármol, se pueden usar para neutralizar la acidez de muchos residuos mineros. Esta corrección de la acidez no sólo favorece el establecimiento de la vegetación, sino que también mitiga la toxicidad al reducir la movilidad de los metales, e incrementa la disponibilidad de nutrientes tales como el potasio, calcio, magnesio, molibdeno o fósforo, que son más móviles a pH superiores a 6 (Barker, 1997). No obstante, incrementos en el pH pueden también estar asociados a incrementos en la movilidad del As, y por tanto es un riesgo existente cuando el As está presente. En la Región de Murcia, la industria de extracción de piedra natural marmórea procesa $147000 \mathrm{~m}^{3}$ de producto al año, generando $128120 \mathrm{t}$ de residuos inertes, de los cuales solo se recuperan un $10 \%$. Este material está constituido en un $98 \%$ por carbonatos, presenta una buena solución para la rehabilitación de materiales ácidos enriquecidos en metales pesados. El uso de enmiendas orgánicas y materiales ricos en carbonatos se ha usado satisfactoriamente para reducir la biodisponibilidad de metales pesados y rehabilitar la función ecológica de los suelos (Pérez de Mora et al., 2005, 2006; Alvarenga et al., 2008; Zornoza et al., 2011)

\section{Objetivos}

El objetivo principal de este estudio es evaluar la eficiencia de la aplicación de un lodo de mármol y purín de cerdo para incrementar la calidad de los suelos y reducir la movilidad de los metales pesados como primera fase en la fitoestabilización asistida de presas de relaves o residuos mineros.

\section{Zona de estudio}

Se ha seleccionado una presa de resiudos mineros denominado Santa Antonieta ( $37^{\circ} 35^{\prime} 38^{\prime \prime} \mathrm{N}, 0^{\circ} 53^{\prime} 11^{\prime \prime}$ W) dentro del distrito minero de Cartagena-La Unión (Región de Murcia, SE España) (Figura 1). Esta zona ha sufrido una minería intensa durante más de 2500 años, aunque la actividad minera finalizó en 1991. El clima del área es Mediterráneo semiárido, con una temperatura media anual de $18^{\circ} \mathrm{C}$ y una precipitación media anual de $275 \mathrm{~mm}$. La evapotranspiración potencial supera los $900 \mathrm{~mm}$ año-1 (Zornoza et al., 2011). La presa tiene una superficie de $14095 \mathrm{~m}^{2}$, y el 


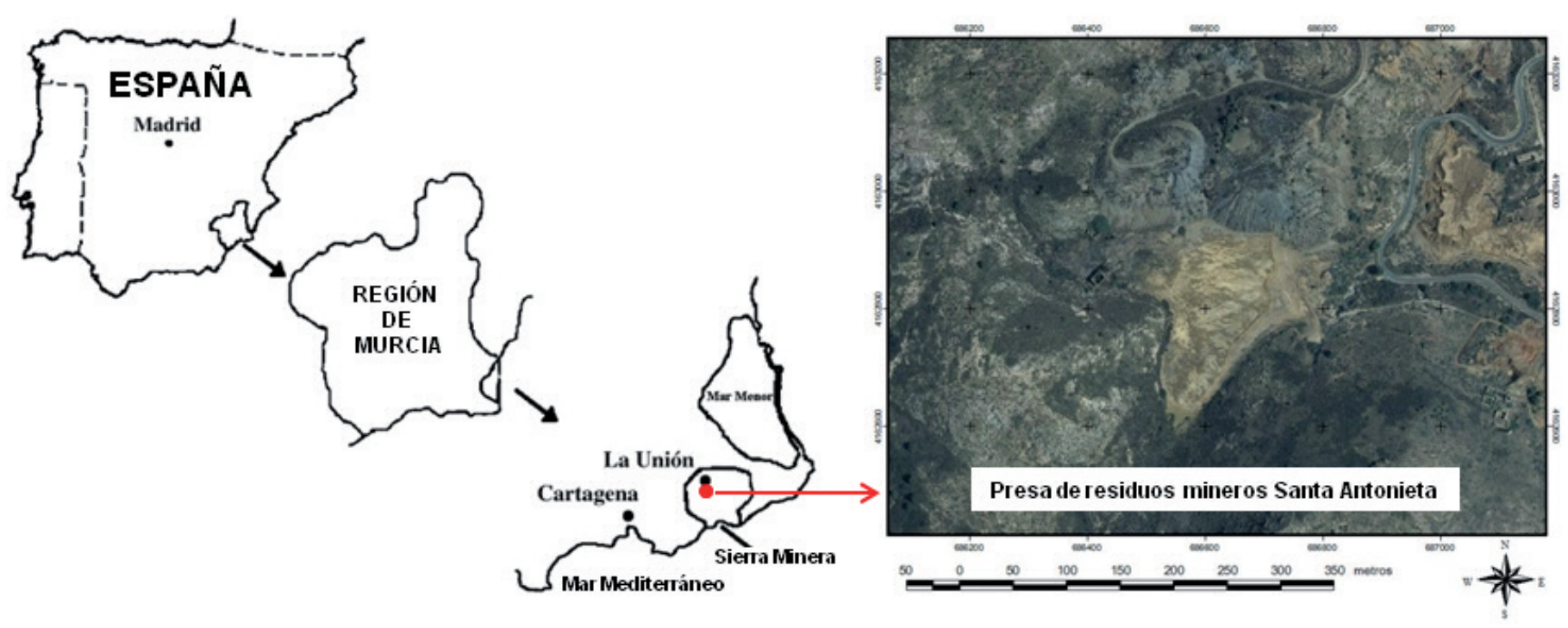

Figura 1. Localización de la zona de estudio.

Figure 1. Location of the study site.

material que lo conforma tiene un $\mathrm{pH}$ medio de 2,9; una textura franco arenosa; inestabilidad y cohesión limitada; bajo contenido en nutrientes y materia orgánica y altas concentraciones de metales pesados. EI rango de concentraciones totales de metales es 0.73 $6.65 \mathrm{mg} \mathrm{kg}^{-1}$ de $\mathrm{Cd}, 1246-2048 \mathrm{mg} \mathrm{kg}^{-1}$ de $\mathrm{Pb}$ y $829-$ $5899 \mathrm{mg} \mathrm{kg}^{-1}$ de $\mathrm{Zn}$. Los niveles de fondo de la zona son de $0.12 \mathrm{mg} \mathrm{kg}^{-1}$ para el $\mathrm{Cd}$, de $9.8 \mathrm{mg} \mathrm{kg}^{-1}$ para el $\mathrm{Pb}$ y de $55 \mathrm{mg} \mathrm{kg}^{-1}$ para el $\mathrm{Zn}$. Los niveles genéricos de referencia son de $0.50 \mathrm{mg} \mathrm{kg}^{-1}$ para el Cd, de $22 \mathrm{mg} \mathrm{kg}^{-}$ ${ }^{1}$ para el $\mathrm{Pb}$ y de $101 \mathrm{mg} \mathrm{kg}^{-1}$ para el Zn (Martínez y Pérez 2007). Como se puede apreciar, los valores de metales encontrados en la presa de residuos mineros de Santa Antonieta exceden estos límites. Según el artículo 2.2.d de la Ley 22/2011, de 28 de julio, de residuos y suelos contaminados, los residuos mineros se excluyen de la aplicación de esta ley y no se consideran legislativamente suelos contaminados. Es el Real Decreto 975/2009 de 12 de junio, sobre gestión de los residuos de las industrias extractivas y de protección y rehabilitación del espacio afectado por actividades mineras, quien regula la gestión de las presas de residuos mineros. El órgano competente minero, basándose en los criterios técnicos establecidos en el Real Decreto 9/2005, de 14 de enero, por el que se establece la relación de actividades potencialmente contaminantes del suelo y los criterios y estándares para la declaración de suelos contaminados, debe asegurar, mediante la caracterización analítica previa, que los residuos mineros no superen los niveles genéricos de referencia para las sustancias contaminantes presentes en el mismo. Si éste no fuera el caso, el órgano minero competente debería responder de ello, ya que tiene competencias en materia de recuperación ambiental en las áreas mineras, y la obligación de velar por la calidad de los suelos (RD 975/2009).

\section{Metodología}

\section{Diseño experimental y muestreo}

Se han utilizado dos enmiendas (purín de cerdo y lodo de mármol $\left(\mathrm{CaCO}_{3}\right)$ ) para rehabilitar la superficie de la presa de residuos mineros, con la finalidad de corregir la acidez, reducir la movilidad de los metales; incrementar el contenido de materia orgánica y de nutrientes; mejorar la estructura del suelo $y$, facilitar el establecimiento y el crecimiento de la vegetación. Las características de las enmiendas empleadas se muestran en la Tabla 1. Para conseguir una correcta aplicación de las enmiendas, se realizó una nivelación de la superficie, y se creó un sistema de drenaje para evitar la formación de zonas encharcadas que pueden comprometer la rehabilitación real de la presa de residuos, además de para el control de posibles lixiviados que se pudieran crear y como medida de protección (Figura 2). Se efectuaron tres aplicaciones de enmienda orgánica, en julio, septiembre y noviembre de 2011, para favorecer la adecuada estabilización de la materia orgánica en el suelo antes del establecimiento de la vegetación. El lodo de mármol se aplicó una sola vez en julio de 2011. Las enmiendas se aplicaron mecánicamente, y todos los materiales 


\begin{tabular}{|c|c|c|c|}
\hline Propiedades & PCB & FSPC & LM \\
\hline $\mathrm{pH}$ & 7,8 & 9,1 & 8,0 \\
\hline Conductividad eléctrica $\left(\mathrm{dS} \mathrm{m}^{-1}\right)$ & 39,1 & 10,2 & 2,2 \\
\hline $\mathrm{CaCO}_{3}(\%)$ & - & - & 98 \\
\hline Humedad (\%) & 96 & 10 & 1 \\
\hline Carbono orgánico total $\left(\mathrm{g} \mathrm{L}^{-1} / \mathrm{g} \mathrm{kg}^{-1}\right)$ & 17,8 & 170,8 & - \\
\hline Nitrógeno total $\left(\mathrm{g} \mathrm{L}^{-1} / \mathrm{g} \mathrm{kg}^{-1}\right)$ & 5,1 & 13,6 & - \\
\hline $\mathrm{C} / \mathrm{N}$ & 3,5 & 12,5 & - \\
\hline $\mathrm{Cu}\left(\mathrm{mg} \mathrm{L}^{-1} / \mathrm{mg} \mathrm{kg}^{-1}\right)$ & 19,30 & 157,35 & 0,36 \\
\hline $\mathrm{Zn}\left(\mathrm{mg} \mathrm{L}^{-1} / \mathrm{mg} \mathrm{kg}^{-1}\right)$ & 28,00 & 732,05 & 0,26 \\
\hline Fósforo disponible ( $\left.\mathrm{mg} \mathrm{L}^{-1} / \mathrm{mg} \mathrm{kg}^{-1}\right)$ & 623 & 9640 & $<$ l.d \\
\hline Calcio ( $\mathrm{mg} \mathrm{L}^{-1} / \mathrm{mg} \mathrm{kg}^{-1}$ ) & 249 & 855 & 2190 \\
\hline Magnesio $\left(\mathrm{mg} \mathrm{L}^{-1} / \mathrm{mg} \mathrm{kg}^{-1}\right)$ & 14,4 & 802 & 347 \\
\hline Sodio $\left(\mathrm{mg} \mathrm{L}^{-1} / \mathrm{mg} \mathrm{kg}^{-1}\right)$ & 459 & 4283 & 69 \\
\hline Potasio $\left(\mathrm{mg} \mathrm{L}^{-1} / \mathrm{mg} \mathrm{kg}^{-1}\right)$ & 1059 & 15662 & 59 \\
\hline
\end{tabular}

Tabla 1. Principales características del purín de cerdo bruto (PCB), la fase sólida del purín de cerdo (FSPC) y el lodo de mármol (LM) usados como enmiendas.

Table 1. Main characteristics of the raw pig slurry $(P C B)$, the solid phase of the pig slurry (FSPC) and the marble mud (LM) used as amendments.

fueron homogeneizados hasta una profundidad de 50 $\mathrm{cm}$ para proceder a una correcta incorporación en el material de superficie de la presa de residuos mineros. El lodo de mármol se aplicó a una dosis de $6.7 \mathrm{~kg}$ $\mathrm{m}^{-2}$, calculado en base a la cantidad de carbonato cálcico necesario para neutralizar la acidez potencial del residuo minero en función de su contenido de sulfuros. Se aplicó un factor de seguridad de 1.5 para asegurar un remanente de carbonato cálcico en el suelo. El contenido total de sulfuros se determinó por oxidación con $\mathrm{H}_{2} \mathrm{O}_{2}$ (Sobek et al., 1978) para establecer la producción de acidez potencial en el suelo como consecuencia de la reacción de los sulfuros (principalmente como pirita, $\mathrm{FeS}_{2}$ ) con el oxígeno atmosférico, un proceso habitual que tiene lugar en la superficie de las acumulaciones de residuos mineros. Se aplicaron $1.7 \mathrm{~L} \mathrm{~m}^{-2}$ de purín de cerdo bruto en julio y $2.6 \mathrm{~L}$ $\mathrm{m}^{-2}$ en septiembre. En noviembre se aplicaron $7 \mathrm{~kg} \mathrm{~m}$ ${ }^{2}$ de la fase sólida de purín de cerdo. La dosis de aplicación del purín bruto de cerdo se basó en la tasa agronómica establecida por el Real Decreto 261/1996 para evitar la contaminación de las aguas subterráneas por nitratos. La dosis de aplicación de la fase sólida del purín se estimó en base a su contenido en carbono orgánico para incrementar el carbono orgánico de la superficie de la presa de residuos mineros $>5 \mathrm{~g}$ $\mathrm{kg}^{-1}$. Esta fase sólida se obtuvo por separación de la fase líquida mediante un separador de fases, y me mantuvo al aire libre bajo condiciones ambientales
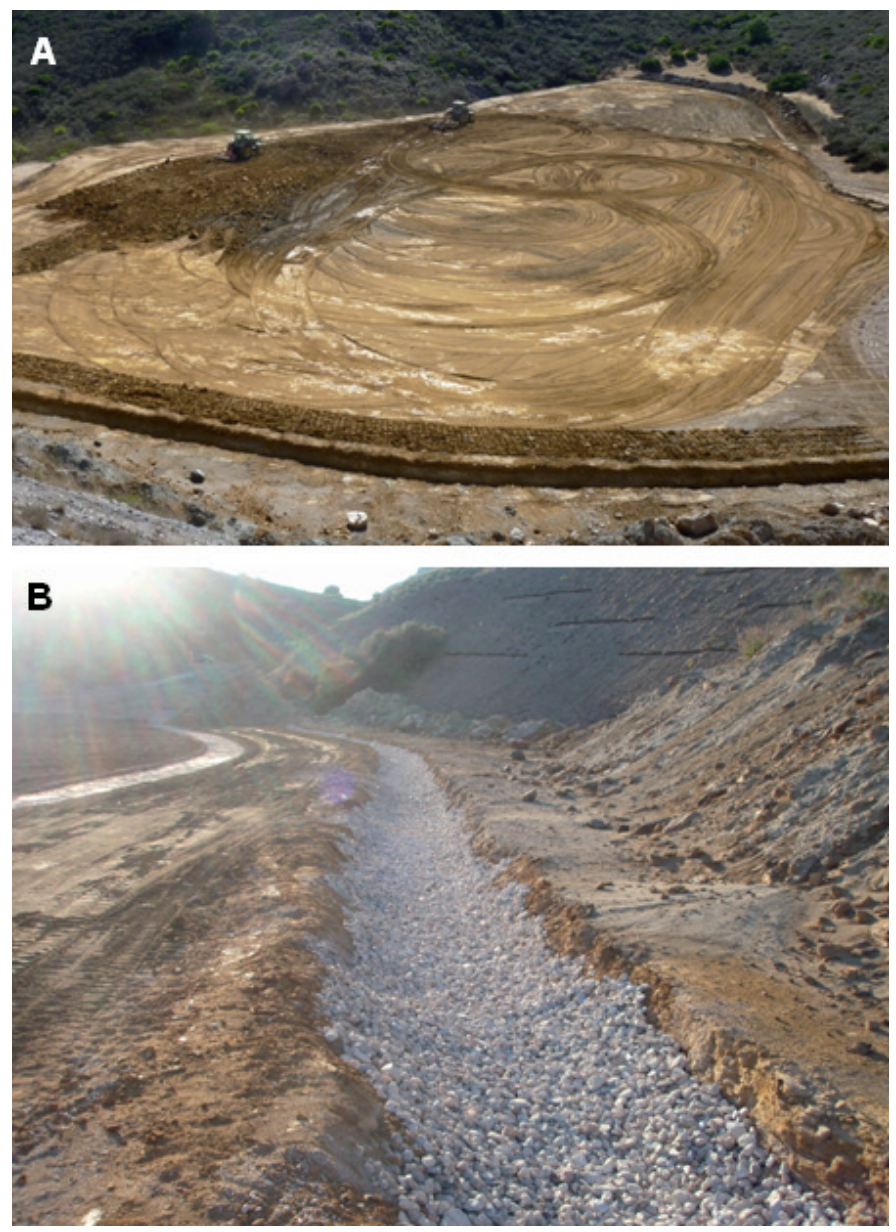

Figura 2. Imágenes de la nivelación de la superficie (A) y de la creación de un sistema de drenaje (B).

Figure 2. Images of the surface levelling $(A)$ and the construction of a drainage system $(B)$.

durante un mes. Entre los meses de marzo y abril de 2012 se llevó a cabo la plantación y siembra de especies autóctonas. Las semillas utilizadas para la siembra directa como para la producción de las plántulas en semilleros se recolectaron de especies que crecían en la zona de estudio Se plantaron con ahoyado manual las siguientes especies en mosaico con un marco de plantación de 1 planta $\mathrm{m}^{-2}$ : Atriplex halimus L., Cistus albidus L., Helichrysum stoechas (L.) Moench, Hyparrhenia hirta (L.) Stapf, Lavandula dentata L., Lygeum spartum (L.) Kunth., Rosmarinus officinalis L. y Phagnalon saxatile (L.) Cass., con un total de 8174 individuos. Se sembraron además las siguientes especies cubriendo toda la superficie de la presa: Cynodon dactylon (L.) Pers., Dittrichia viscosa (L.) Greuter, Piptatherum miliaceum (L.) Coss, Sonchus tenerrimus L. y Limonium caesium (Girard.) Kuntze, con un total de $23240 \mathrm{~g}$ de semillas. 
Para llevar a cabo un seguimiento de la evolución de la calidad del suelo, se establecieron cuatro parcelas experimentales de $10 \mathrm{~m} \times 10 \mathrm{~m}$ distribuidas por la superficie de la presa de residuos mineros. Se llevó a cabo un muestreo inicial en octubre 2010, y cuatro muestreos semestrales en enero y julio 2012 y en enero y julio 2013, iniciando estos muestreos de seguimiento seis meses después de la primera aplicación de las enmiendas. En cada muestreo se tomaron tres muestras de suelo por parcela, a dos profundidades diferentes: $0-15$ y $15-30 \mathrm{~cm}$ (Figura 3). Las muestras se llevaron al laboratorio, se secaron al aire durante una semana y se tamizaron a un tamaño $<2$ $\mathrm{mm}$.

\section{Métodos analíticos}

El pH y la conductividad eléctrica (CE) se midieron en agua desionizada (relación 1:2.5 y 1:5 p/v, respectivamente); la textura se determinó usando el método de la pipeta de Robinson combinado con tamizado; la estabilidad de agregados (EA) se determinó mediante el método propuesta por USDA (1999); el carbono orgánico total (Corg), el carbono inorgánico y el nitrógeno total $(\mathrm{Nt})$ se determinaron con un analizador elemental CNHS-O (EA-1108, Carlo Elba); el contenido en carbonato cálcico se estimó a partir del contenido en carbono inorgánico; la capacidad de intercambio catiónico $(\mathrm{CIC})$ se midió con el método de Chapman (1965); el contenido total de los metales pesados (Cd, $\mathrm{Pb}$ y $\mathrm{Zn}$ ) se determinó mediante una digestión con $\mathrm{HNO}_{3} / \mathrm{HClO}_{4}$ a $210^{\circ} \mathrm{C}$ durante $1.5 \mathrm{~h}$ (Risser y Baker, 1990). La fracción biodisponible de los metales pesados se extrajo con $\mathrm{CaCl}_{2}$ 0,01 $\mathrm{M}$ (relación 1:10 p/v) (Pueyo et al., 2004). La concentración de los metales pesados se midió en ICP-MS (Agilent $7500 \mathrm{CE}$ ). siendo los límites de detección del equipo $0,02 \mu \mathrm{g} \mathrm{L}^{-1}$ para el Cd; 0,35 $\mu \mathrm{g} \mathrm{L}^{-1}$ para el $\mathrm{Pb}$ y $0.96 \mu \mathrm{g} \mathrm{L}^{-1}$ para el $\mathrm{Zn}$. El material certificado de referencia usado en las medidas de concentración total de metales, corresponde a un suelo referencia siendo éste el denominado BAMU110 (Federal Institute for Materials Research and Testing, Alemania). Los ratios de recuperación fueron $96-126 \%$ para el $\mathrm{Cd}, 95-106 \%$ para el $\mathrm{Pb}$ y $82-98 \%$ para el Zn.

\section{Análisis estadístico}

El ajuste de los datos a una distribución normal para todas las propiedades se verificó con el test Kolmogorov-Smirnov. Se llevó a cabo un ANOVA de un factor para evaluar las diferencias encontradas en
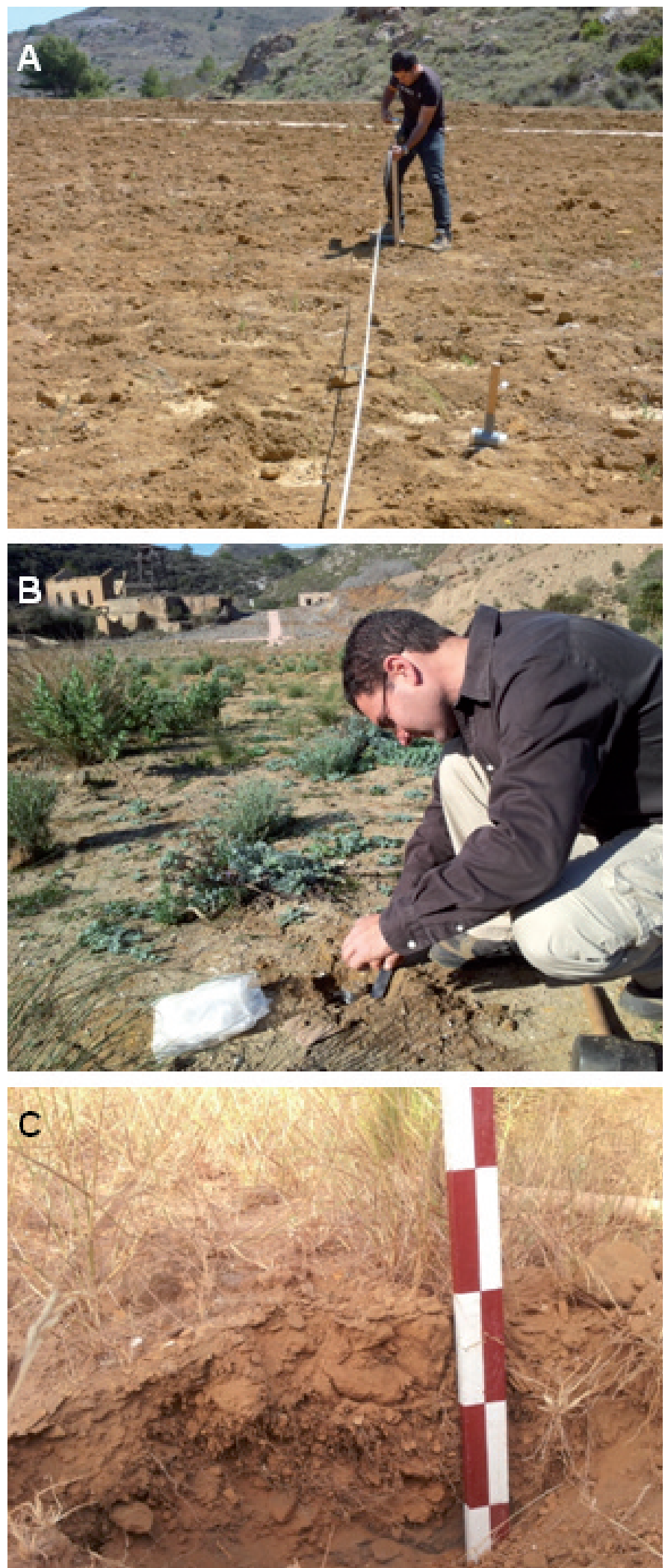

Figura 3. Imágenes del establecimiento de las parcelas experimentales (A), muestreo de suelo (B) y perfil de suelo enmendado (C). Figure 3. Images of the establishment of the experimental plots $(A)$, soil sampling $(B)$ and amended soil profile $(C)$. 
función del tiempo de muestreo y de la profundidad. La separación de las medias se efectuó según el test post-hoc de Tukey a un nivel de significación $\mathrm{P}<0.05$. Para identificar relaciones entre las diferentes propiedades se realizaron correlaciones Pearson. El análisis estadístico se llevó a cabo con el programa IBM SPSS para Windows, versión 19 (2010).

\section{Resultados y discusión}

\section{Propiedades físico-químicas}

La aplicación del lodo de mármol y el purín de cerdo a los residuos mineros ha provocado cambios en sus propiedades que se han mantenido durante los dos años de ejecución de este estudio. El pH se incrementó tras la aplicación de las enmiendas desde valores cercanos a 3 (2.89-3.12) hasta valores cercanos a la neutralidad, y se ha mantenido sin diferencias significativas a lo largo del periodo de estudio (Figura 4a). Este incremento es debido a la presencia de carbonato cálcico en los suelos, que es capaz mantener el pH próximo a la neutralidad durante largo tiempo. La correlación significativa positiva entre el $\mathrm{pH}$ y el contenido de $\mathrm{CaCO}_{3}$ apoya esta relación $(\mathrm{r}=0.68$; $\mathrm{P}<0.001)$. Fernández-Caliani y Barba-Brioso (2010) y Kabas et al. (2013) también observaron que una única aplicación de lodo de mármol incrementaba el pH de residuos mineros ácidos hasta la neutralidad, manteniéndose estable durante al menos un año. El lodo de mármol usado como enmienda es alcalino, con un $\mathrm{pH}$ de 8 y formado en un $98 \%$ por carbonato cálcico (Tabla 1), siendo un material altamente efectivo para neutralizar la acidez. Además, este lodo está formado por partículas muy finas $(5-10 \mu \mathrm{m})$ con alta superficie específica, lo que favorece su uso y efectividad como enmienda para alcalinizar el medio. La neutralización
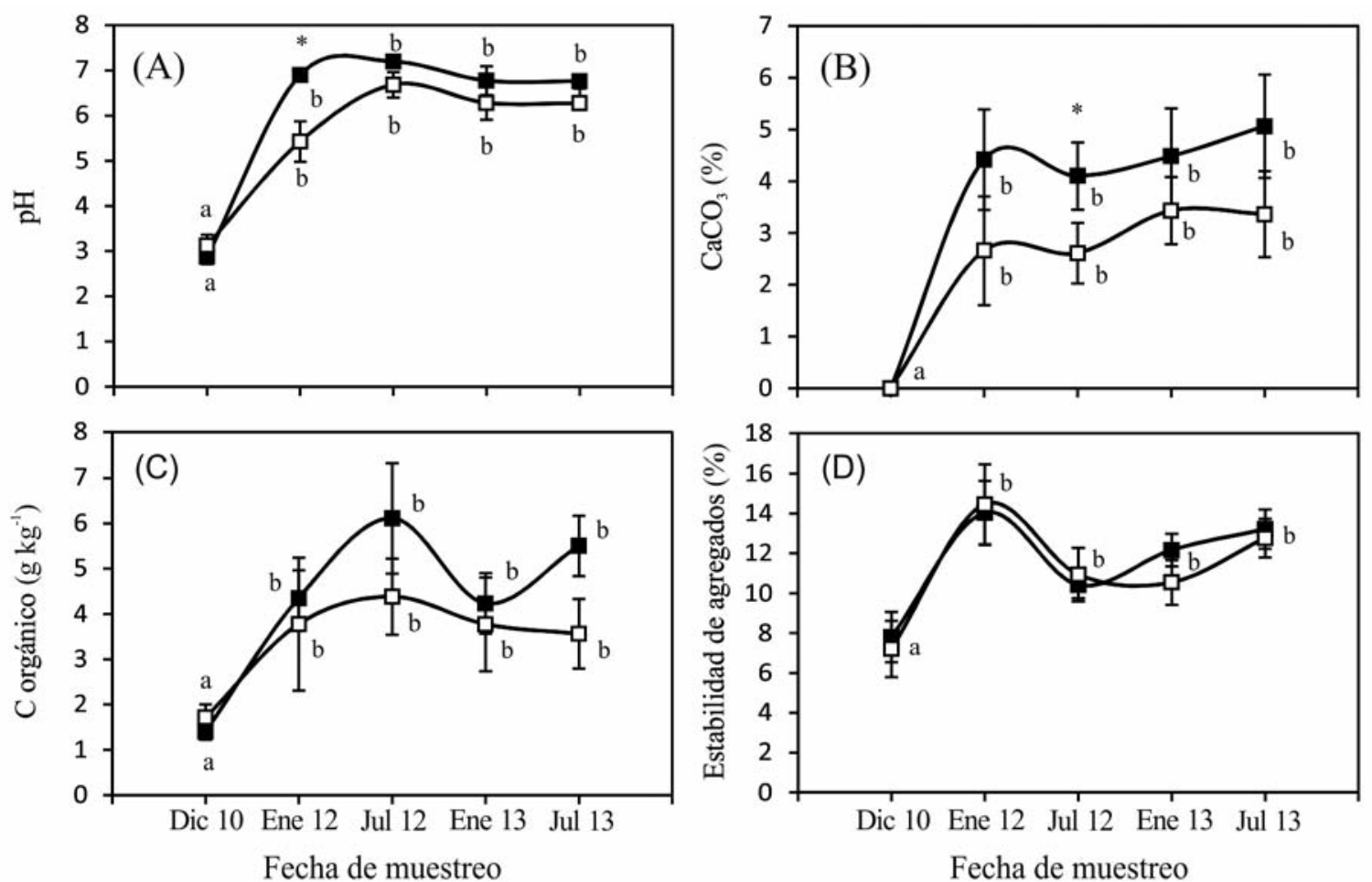

Figura 4. Evolución temporal del pH (A), carbonato cálcico (B), carbono orgánico (C) y estabilidad de agregados (D) en las muestras de suelo de la presa de residuos mineros de Santa Antonieta. La aplicación de enmiendas tuvo lugar entre julio y noviembre de 2011 , y la plantación/siembra entre marzo y abril de 2012. Las barras verticales indican el error típico. Las letras diferentes indican diferencias significativas entre fechas de muestreo para una misma profundidad tras la realización del test de Tukey a $P<0,05$. Los asteriscos $(*)$ indican diferencias significativas entre muestras superficiales y supsuperficiales para una misma fecha de muestreo. Los símbolos negros representan las muestras superficiales $(0-15 \mathrm{~cm})$ mientras que los símbolos blancos representan las muestras supsuperficiales (15-30 $\mathrm{cm})$.

Figure 4. Temporary evolution of $\mathrm{pH}(A)$, calcium carbonate $(B)$, organic carbon $(C)$ and aggregate stability $(D)$ in the samples collected from the Santa Antonieta tailings pond. The application of amendments took place between July and November 2011, and the planting/seeding between March and April 2012. Vertical bars represent the typical error. Different letters indicate significant differences among sampling dates for the same depth afterTukey's $(P<0.05)$. Asterisks $\left(^{*}\right)$ indicate significant differences between surface and sub-surface samples for the same sampling date. Black symbols represent surface samples $(0-15 \mathrm{~cm})$ and white symbols represent sub-surface samples $(15-30 \mathrm{~cm})$. 
de la acidez se debe a la disolución de la calcita $\left(\mathrm{CaCO}_{3}\right)$ a través de las siguientes reacciones (Fernández-Caliani y Barba-Brioso, 2010):

$$
\begin{gathered}
\mathrm{CaCO}_{3}+\mathrm{H}_{2} \mathrm{CO}_{3}=\mathrm{Ca}^{+2}+2 \mathrm{HCO}_{3}^{-} \\
\mathrm{CaCO}_{3}+\mathrm{H}_{2} \mathrm{O}=\mathrm{Ca}^{+2}+\mathrm{HCO}_{3}^{-}+\mathrm{OH}^{-}
\end{gathered}
$$

El rango de $\mathrm{pH}$ alcanzado con la aplicación del lodo de mármol en los suelos coincide con el rango efectivo de precipitación y adsorción de la mayoría de metales pesados (McLean y Bledsoe, 1992), lo que lo convierte en una enmienda adecuada para la rehabilitación de suelos mineros donde el As no sea el contaminante mayoritario. En el muestreo de enero 2012, justo seis meses tras la aplicación de las enmiendas, el $\mathrm{pH}$ en las muestras sub-superficiales fue de 5.4 y significativamente inferior al valor en superficie (6.9), aunque con el tiempo el valor del $\mathrm{pH}$ en supsuperficie se incrementó a valores similares a la superficie. Esto puede ser debido al diferente contenido de carbonato cálcico en función de la profundidad, siendo significativamente inferior en supsuperficie (Figura 4b), por lo que fue necesario más tiempo para que se diera la neutralización de la acidez del residuo minero. Estas diferencias en el contenido de carbonato cálcico con la profundidad pueden deberse a que la incorporación de las enmiendas en los $50 \mathrm{~cm}$ superficiales de la presa de residuos mineros no fue del todo homogénea, ya que la aplicación fue superficial y la incorporación se realizó de forma mecánica. De este modo, la concentración de carbonato cálcico en superficie ascendió de 0 a un máximo de $5.10 \%$ en superficie, mientras que solo ascendió hasta 2.61-3.43 en supsuperficie. No obstante, estos niveles han contribuido a la neutralización de la acidez de los residuos mineros a ambas profundidades, manteniéndose estables con el tiempo. La presencia de carbonatos libres en el medio asegura que el suelo rehabilitado tiene capacidad para neutralizar la acidez que pueda generar la oxidación de los sulfuros presentes, fundamentalmente la pirita. No obstante, parte de las partículas de carbonato cálcico presentes en el suelo podrían estar revestidas por precipitados de óxidos e hidróxidos de hierro, aluminio y manganeso (Simón et al., 2005), perdiendo su reactividad. Por este motivo se aplicó el factor de seguridad de 1.5 a la hora de calcular la dosis de aplicación del lodo de mármol, para garantizar un remanente de carbonato cálcico libre.

El contenido de Corg se encontraba en torno a 1.5 $\mathrm{g} \mathrm{kg}^{-1}$ antes de la aplicación de las enmiendas, y significativamente ascendió a valores promedio superiores a $3.5 \mathrm{~g} \mathrm{~kg}^{-1}$ como consecuencia de la adición del purín de cerdo, fundamentalmente en su fase sólida (Figura 4c). El contenido en Corg se ha mantenido relativamente estable durante los dos años de duración de este estudio, sin diferencias significativas entre muestreos. Aunque el nivel medio de este parámetro es ligeramente superior en superficie, no existen diferencias significativas con las muestras tomadas a $15-30 \mathrm{~cm}$. Incrementos en el contenido de materia orgánica son esenciales para garantizar una rehabilitación real de los suelos, ya que es una propiedad íntimamente relacionada con las mejoras en la estructura del suelo, aireación, retención y circulación de agua, reducción de las tasas de erosión, estimulación de las poblaciones microbianas y en incrementos en la fertilidad por liberación de nutrientes (Smith et al., 1993; Bukert et al., 2000; Shafi et al., 2007). La tendencia habitual del Corg, sobre todo en ambientes áridos y semiáridos, es la mineralización, de manera que una sola aplicación de estiércol porcino debería conducir a una ligera reducción en el contenido de Corg del suelo a medio y largo plazo (Zornoza et al., 2012). El mantenimiento de los niveles de Corg durante dos años puede deberse por tanto al establecimiento de la vegetación, que se ha desarrollado con éxito en la superficie de la presa de residuos mineros, contribuyendo con nuevos aportes de materia orgánica a través de los exudados radiculares y por acumulación de hojarasca, lo que garantiza la rehabilitación real de la presa de residuos mineros (Bouwman y Vangronsveld, 2004). De hecho, la superficie de la presa de residuos estaba desprovista de vegetación, y tras dos años de estudio la cubierta vegetal ascendió al $65 \%$.

La EA ha sido muy similar en las muestras superficiales y sub-superficiales, sin diferencias significativas entre ellas, con un aumento significativo en sus valores tras la aplicación de las enmiendas (Figura 4d). La EA se ha mantenido relativamente estable con el tiempo, sin diferencias significativas entre los diferentes muestreos, con valores medios entre 10.4 y $14.5 \%$. Este incremento en la agregación de las partículas minerales se debe a la incorporación de materia orgánica y carbonato cálcico con las enmiendas que contribuyen a la formación de nuevos agregados, que ayudan a incrementar la porosidad, la capacidad de retención hídrica, el desarrollo y establecimiento de la vegetación, el desarrollo de las comunidades microbianas, el movimiento animal y la reducción de la erosión (Six et al., 2004). La presencia del ion calcio procedente del lodo de mármol puede favorecer las uniones entre los minerales de la arcilla, y promo- 
ver interacciones intermoleculares entre los compuestos orgánicos e inorgánicos, formando agregados (Baldock y Skjemstad, 2000; Clough y Skjemstad, 2000). Era de esperar una correlación significativa positiva entre la EA y el contenido en Corg y $\mathrm{CaCO}_{3}$, pero no se ha observado. Esto puede sugerir que la agregación podría estar más asociada a cambios en la fracción mineral que en la materia orgánica.

Un efecto observado tras la aplicación de las enmiendas fue el descenso significativo de la CE, que se mantuvo inferior a los niveles iniciales durante los dos años de estudio (Figura 5a). Este descenso fue significativamente más acusado en las muestras superficiales. Esto podría ser debido a la aplicación del lodo de mármol, ya que su concentración también era mayor en la superficie. De hecho existe una correlación negativa significativa entre la $\mathrm{CE}$ y el contenido en $\mathrm{CaCO}_{3}(\mathrm{r}=-0.58, \mathrm{P}<0.001)$. Esto podría ser debido a la reacción del $\mathrm{Ca}^{+2}$ con los sulfatos procedentes de la oxidación de los sulfuros como la pirita, la blenda, la galena, etc, formando precipitados minerales que descienden el contenido de sulfatos en la disolución del suelo. Fernández-Caliani y Barba-Brioso (2010) explicaron que es habitual la neoformación de cristales de yeso en suelos mineros enmendados por la precipitación a partir de la solución saturada en sulfato de calcio según la reacción:

$$
\mathrm{Ca}^{+2}+\mathrm{SO}_{4}^{-2}+2 \mathrm{H}_{2} \mathrm{O} \gg>\mathrm{CaSO}_{4} 2 \mathrm{H}_{2} \mathrm{O}
$$

Por otro lado, el incremento de $\mathrm{pH}$ favorece la precipitación de diferentes iones en el medio (Brallier et al., 1996), que también ha podido contribuir a descensos en la CE. La correlación negativa entre la CE y el $\mathrm{pH}$ apoya esta hipótesis $(r=-0.52 ; \mathrm{P}<0.001)$. Estos descensos en la CE son muy positivos, ya que estos materiales son altamente salinos, lo que podría comprometer el correcto desarrollo de la vegetación, u obligaría al uso de especies halófitas para crear una cubierta vegetal.

Aunque antes de la aplicación de las enmiendas el contenido en $\mathrm{Nt}$ era muy bajo y similar entre las muestras superficiales y supsuperficiales $(\sim 0.35 \mathrm{~g} \mathrm{~kg}$ 1), tras la aplicación de las enmiendas el Nt se incrementó significativamente, siendo este incremento más acusado en las muestras superficiales (Figura 5b). Zanuzzi et al. (2009) también observaron incrementos en el Nt de la presa de residuos mineros tras la aplicación de estiércol de cerdo como enmienda. Con este parámetro se ha observado una tendencia creciente hasta julio de 2012 en superficie $(0.91 \mathrm{~g} \mathrm{~kg}$ 1) y hasta enero enero de 2012 en supsuperficie (0.59 $\mathrm{g} \mathrm{kg}^{-1}$ ), para iniciar una tendencia decreciente. Las
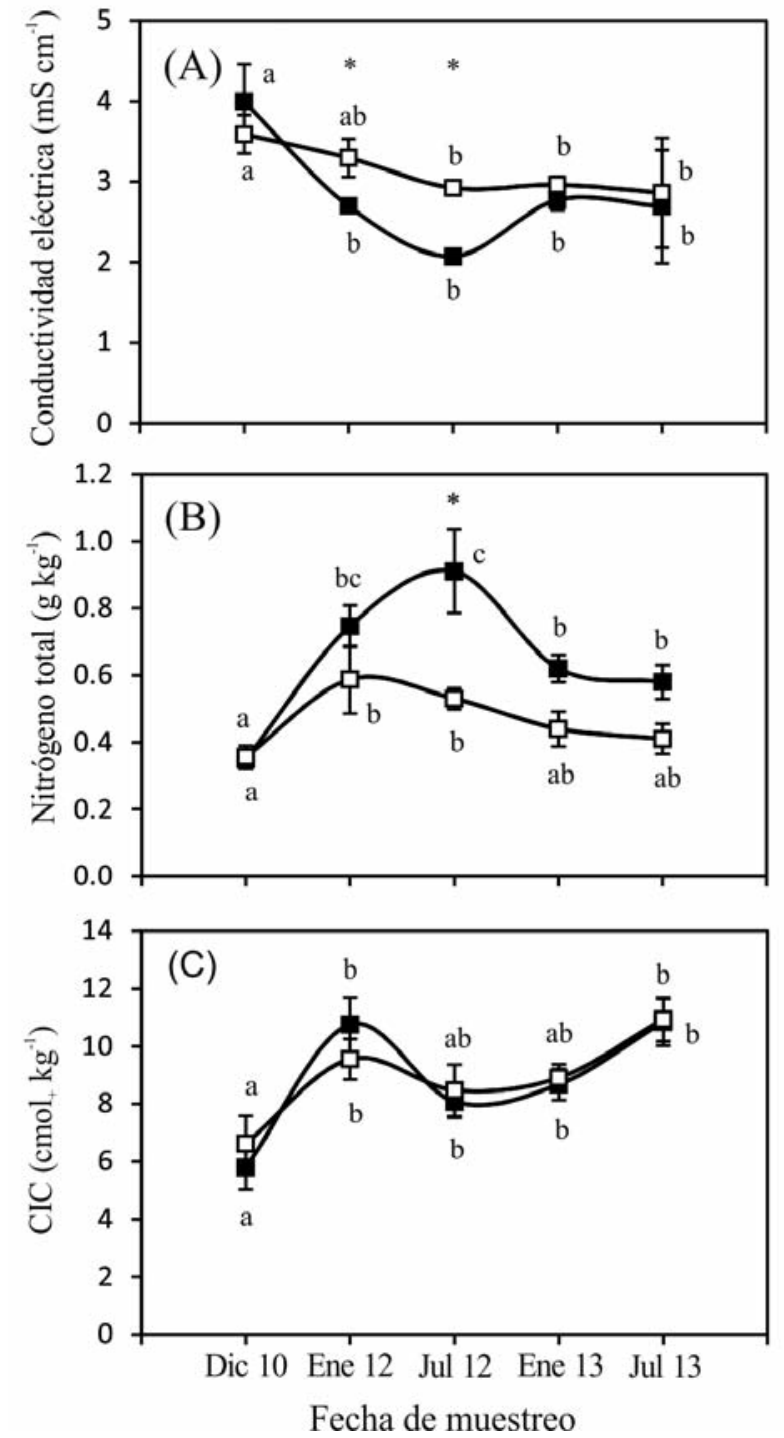

Figura 5. Evolución temporal de la conductividad eléctrica (A), nitrógeno total $(B)$ y capacidad de intercambio catiónico $(C)$ en las muestras de suelo de la presa de residuos mineros de Santa Antonieta. La aplicación de enmiendas tuvo lugar entre julio y noviembre de 2011, y la plantación/siembra entre marzo y abril de 2012. Las barras verticales indican el error típico. Las letras diferentes indican diferencias significativas entre fechas de muestreo para una misma profundidad tras la realización del test de Tukey a $P<0.05$. Los asteriscos $(*)$ indican diferencias significativas entre muestras superficiales y supsuperficiales para una misma fecha de muestreo. Los símbolos negros representan las muestras superficiales $(0-15 \mathrm{~cm})$ mientras que los símbolos blancos representan las muestras supsuperficiales $(15-30 \mathrm{~cm})$.

Figure 5. Temporary evolution of electrical conductivity $(A)$, total nitrogen $(B)$ and cation exchange capacity $(C)$ in the samples collected from the Santa Antonieta tailings pond. The application of amendments took place between July and November 2011, and the planting/seeding between March and April 2012. Vertical bars represent the typical error. Different letters indicate significant differences among sampling dates for the same depth after Tukey's $(P<0.05)$. Asterisks $\left(^{*}\right)$ indicate significant differences between surface and sub-surface samples for the same sampling date. Black symbols represent surface samples $(0-15 \mathrm{~cm})$ and white symbols represent sub-surface samples $(15-30 \mathrm{~cm})$. 
muestras supsuperficiales en profundidad en el último muestreo de julio 2013 presentan un contenido de $\mathrm{Nt}$ sin diferencias significativas con el contenido inicial. El hecho de que las muestras superficiales presenten mayor concentración de Nt puede estar indicando probablemente que la incorporación del purín de cerdo en los primeros $50 \mathrm{~cm}$ de la superficie de la presa de residuos mineros no fue completa, sin una perfecta homogenización con el residuo minero. EI incremento del contenido de este macronutriente es muy positivo ya que el $\mathrm{N}$ es un elemento esencial para la vegetación y para las comunidades microbianas. Por tanto, el aumento inicial contribuyó a la mejora de las condiciones nutricionales suelo y al crecimiento y desarrollo de las plantas introducidas. EI descenso observado en $\mathrm{Nt}$ con el tiempo puede estar indicando una absorción de este nutriente por parte de la planta, e inmovilización en forma de biomasa microbiana. Para evitar que la falta de Nt pueda limitar el desarrollo de la vegetación, sería interesante incluir leguminosas entre las especies introducidas en la presa de residuos mineros, con el fin de incrementar la disponibilidad de este nutriente mediante fijación biológica de $\mathrm{N}$ atmosférico.

La CIC se incrementó significativamente tras la aplicación de las enmiendas, manteniéndose estos valores sin diferencias significativas entre los diferentes muestreos (Figura 5b). La aplicación de la fase sólida del purín de cerdo contribuyó positivamente al incremento de este indicador de fertilidad edáfica, debido al incremento en compuestos orgánicos con grupos orgánicos funcionales como carboxilos $(-\mathrm{COOH})$ e hidroxilo $(-\mathrm{OH})$ que se desprotonan a $\mathrm{pH}$ neutro y constituyen nuevas posiciones de cambio para cationes.

\section{Metales pesados}

La fracción biodisponible de todos los metales pesados analizados extraída con $\mathrm{CaCl}_{2}$ descendió significativamente con la aplicación de las enmiendas, y se mantuvo estable durante el último año de estudio (Figura 6). En superficie, el Cd descendió de $390 \mu \mathrm{g}$ $\mathrm{kg}^{-1}$ antes de la aplicación de las enmiendas a valores $<39 \mu \mathrm{g} \mathrm{kg}^{-1}$ tras la aplicación de las enmiendas (descenso del $90 \%$ ), sin diferencias significativas entre los cuatro muestreos realizados (Figura 6a). En las muestras supsuperficiales, el Cd descendió desde $590 \mu \mathrm{g}$ $\mathrm{kg}^{-1}$ hasta $420 \mu \mathrm{g} \mathrm{kg}^{-1}$ seis meses tras la aplicación de las enmiendas, y continuó esta tendencia decreciente en el siguiente muestreo con una concentración de 52 $\mu \mathrm{g} \mathrm{kg}{ }^{-1}$, que se mantuvo estadísticamente estable hasta el final del estudio (descenso del $91 \%$ ). No hubo
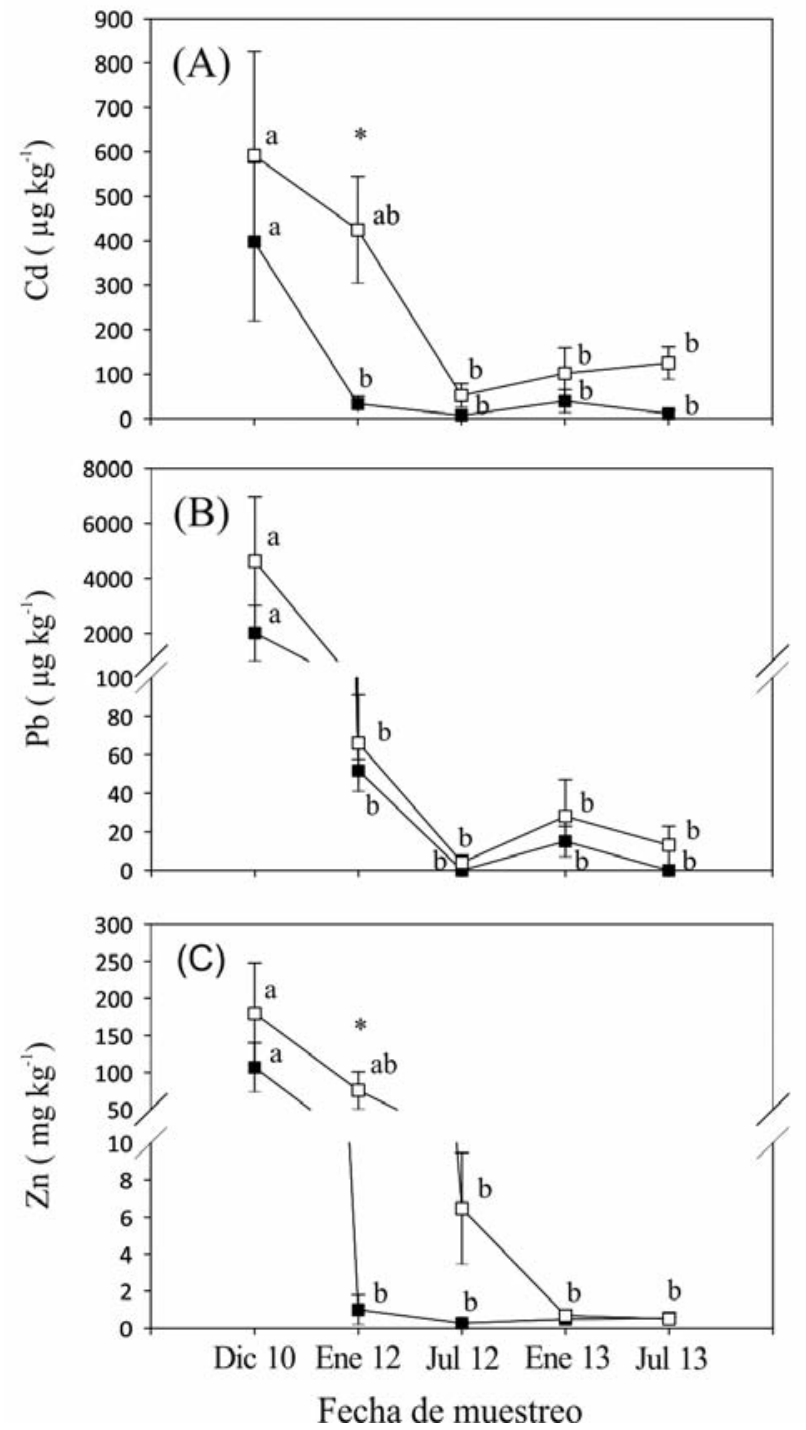

Figura 6. Evolución temporal de la fracción biodisponible de cadmio (A), plomo (B) y zinc (C) en las muestras de suelo de la presa de residuos mineros de Santa Antonieta. La aplicación de enmiendas tuvo lugar entre julio y noviembre de 2011, y la plantación/siembra entre marzo y abril de 2012. Las barras verticales indican el error típico. Las letras diferentes indican diferencias significativas entre fechas de muestreo para una misma profundidad tras la realización del test deTukey a $P<0.05$. Los asteriscos $(*)$ indican diferencias significativas entre muestras superficiales $y$ supsuperficiales para una misma fecha de muestreo. Los símbolos negros representan las muestras superficiales $(0-15 \mathrm{~cm})$ mientras que los símbolos blancos representan las muestras supsuperficiales $(15-30 \mathrm{~cm})$.

Figure 6. Temporary evolution of the bio-available fraction of cadmium $(A)$, lead $(B)$ and zinc $(C)$ in the samples collected from the Santa Antonieta tailings pond. The application of amendments took place between July and November 2011, and the planting/seeding between March and April 2012. Vertical bars represent the typical error. Different letters indicate significant differences among sampling dates for the same depth after Tukey's $(P<0.05)$. Asterisks (*) indicate significant differences between surface and sub-surface samples for the same sampling date. Black symbols represent surface samples $(0-15 \mathrm{~cm})$ and white symbols represent sub-surface samples $(15-30 \mathrm{~cm})$. 
diferencias significativas entre ambas profundidades muestreadas excepto en el primer muestreo tras la aplicación de las enmiendas (enero 2012), con mayor concentración en las muestras supsuperficiales. La fracción biodisponible del $\mathrm{Pb}$ descendió significativamente de una concentración de $2019 \mu \mathrm{g} \mathrm{kg}^{-1}$ hasta valores $<50 \mu \mathrm{g} \mathrm{kg}^{-1}$ tras la aplicación de las enmiendas (descenso del 97\%), sin diferencias significativas entre los diferentes muestreos de seguimiento. Las muestras supsuperficiales siguieron la misma tendencia, sin diferencias significativas entre ambas profundidades, con concentraciones de $\mathrm{Pb}$ biodisponible que descendieron significativamente de $4627 \mathrm{\mu g} \mathrm{kg}^{-1} \mathrm{a}$ valores $<66 \mathrm{\mu g} \mathrm{kg}^{-1}$ (Figura 6b). La concentración de $\mathrm{Zn}$ biodisponible descendió significativamente de $107.28 \mathrm{mg} \mathrm{kg}^{-1}$ a valores $<0.99 \mathrm{mg} \mathrm{kg}^{-1}$ tras la aplicación de las enmiendas (descenso del 99\%), sin diferencias significativas entre los diferentes muestreos de seguimiento (Figura 6c). En las muestras supsuperficiales, el $\mathrm{Zn}$ descendió desde $180.28 \mathrm{mg} \mathrm{kg}^{-1}$ hasta $76.76 \mathrm{mg} \mathrm{kg}^{-1}$ seis meses tras la aplicación de las enmiendas, y continuó esta tendencia decreciente en los siguientes muestreos con una concentración de $6.47 \mathrm{mg} \mathrm{kg}^{-1}$ en julio 2012 y $<0.70 \mathrm{mg} \mathrm{kg}^{-1}$ en enero y julio 2013 (descenso del 99\%). No hubo diferencias significativas entre ambas profundidades muestreadas excepto en el primer muestreo tras la aplicación de las enmiendas (enero 2012), con mayor concentración en las muestras supsuperficiales.

Si tenemos en cuenta el porcentaje del contenido total de cada metal que es biodisponible, observamos que con respecto al $\mathrm{Cd}$, éste presentaba un $11.4 \%$ y $17.1 \%$ de biodisponibilidad al inicio del ensayo, para las muestras superficiales y supsuperficiales, respectivamente. Al final del estudio, la biodisponibilidad del Cd descendió hasta el $0.6 \%$ y $2.9 \%$ para muestras superficiales y supsuperficiales, respectivamente.

$\mathrm{El} \mathrm{Pb}$, que es un metal que presenta muy baja solubilidad, mostró una fracción biodisponible al inicio del estudio del $0.12 \%$ en superficie y del $0.31 \%$ en supsuperficie. Con la adición de las enmiendas la fracción biodisponible descendió hasta el $0.00 \%$ del contenido total. El Zn presentó una fracción biodisponible del $2.8 \%$ y $5.7 \%$ para las muestras superficiales y supsuperficiales, respectivamente. Con la adición de las enmiendas la biodisponibilidad descendió hasta el $0,03 \%$ en todas las muestras.

El descenso en la concentración de la fracción biodisponible de los metales pesados se debe fundamentalmente a un efecto directo del incremento del $\mathrm{pH}$, que favorece los procesos de adsorción, precipitación y coprecipitación con oxihidróxidos, a la formación de quelatos con la materia orgánica, a la formación de carbonatos metálicos (aportados por el lodo de mármol) y a la formación de fosfatos metálicos (aportados por el purín de cerdo) como consecuencia de la aplicación de las enmiendas (Alvarenga et al., 2008; Liu et al., 2009; Fernández-Caliani y Barba-Brioso, 2010; Zornoza et al., 2013). Zornoza et al. (2013) observaron que la formación de complejos orgánicos y la precipitación como fosfato eran procesos muy efectivos la para la inmovilización del Cd, mientras que la adición de carbonatos era necesaria para reducir la biodisponibilidad del $\mathrm{Pb}$ y del $\mathrm{Zn}$.

Las diferencias observadas entre las muestras superficiales y subsuperficiales en la concentración de $\mathrm{Cd}$ y $\mathrm{Zn}$ biodisponibles en el primer muestreo tras la aplicación de las enmiendas (enero 2012), puede ser debido a los menores valores de $\mathrm{pH}$ en este muestreo $(5,2$ en supsuperficie frente a 6,9 en superficie) como consecuencia del menor contenido de carbonato cálcico, por lo que ha sido necesario más tiempo para neutralizar la acidez hasta niveles similares a las muestras superficiales. Existe una correlación significativa negativa entre el $\mathrm{pH}$ y el $\mathrm{Cd}(\mathrm{r}=-0.57 ; \mathrm{P}<0.001)$ y el $Z n(r=-0.59 ; P<0.001)$, que apoya esta hipótesis. Por otro lado, la vegetación puede también haber contribuido a la inmovilización de los metales, ya que se introdujo entre los meses de marzo y abril de 2012. La vegetación puede contribuir a la estabilización a largo plazo de suelos mineros mediante la creación de un ambiente rizosférico mediante los procesos que ocurren en la rizosfera, que favorecen la precipitación y estabilización de los metales pesados (Méndez y Maier, 2008). Por otro lado, Kabas et al. (2012) observaron que el descenso de la disponibilidad de $\mathrm{Cd}, \mathrm{Cu}$ y $\mathrm{Pb}$, en una presa de residuos mineros, era mayor cuando estaban presentes las especies Atriplex halimus, Piptatherum miliaceum y Zigophyllum fabago en comparación con la aplicación de enmiendas sin la presencia de vegetación, poniendo de manifiesto la necesidad de favorecer la creación de una cubierta vegetal para garantizar la estabilización de metales pesados en los suelos.

Estos resultados confirman la idoneidad del lodo de mármol y el purín de cerdo, aplicados en bruto y en su fase sólida, para la rehabilitación de residuos mineros ácidos con altas concentraciones de metales pesados y ausencia de nutrientes, que facilitan las condiciones necesarias para el establecimiento de una cubierta vegetal capaz de inmovilizar los metales pesados y frenar la erosión del suelo. Pardo et al. (2011) también concluyeron que el uso de purín de cerdo tenía efectos positivos en la rehabilitación de suelos mineros, mejorando las propiedades del suelo e incrementando la germinación de semillas introducidas. Zornoza et al. (2013) observaron además que la adición de carbonato cálcico en residuos mineros 
rehabilitados estabiliza el carbono orgánico aportado por las enmiendas orgánicas, minimizando las pérdidas de materia orgánica en el suelo por mineralización. Por tanto, el lodo de mármol además de provocar incrementos en el pH e inmovilización de metales pesados, también favorece la acumulación de materia orgánica en el suelo. Estos resultados son de especial interés en regiones donde el manejo de los purines de cerdo y los residuos de la actividad extractiva de material carbonatado representan un problema medioambiental debido a las grandes cantidades producidas. No obstante se propone una monitorización a más largo plazo para evaluar la eficacia de las distintas especies vegetales utilizadas, los posibles efectos de los contaminantes en ellas, ysu distribución en los tejidos vegetales.

\section{Conclusiones}

La estrategia de rehabilitación de presas de residuos mineros desarrollada basada en la fitoestabilización asistida con lodo de mármol y purín de cerdo ha resultado positiva en la mejora de la calidad del suelo y descenso de la biodisponibilidad de los metales pesados. Se ha incrementado significativamente el $\mathrm{pH}$ hasta valores cercanos a la neutralidad, con incrementos en el carbono orgánico, en la estabilidad de agregados, en el nitrógeno total y en la capacidad de intercambio catiónico, con descensos en la conductividad eléctrica. La fracción biodisponible de los metales pesados ha descendido hasta un $90-99 \%$. Estos resultados son de gran interés en áreas con actividad extractiva de material carbonatado (caliza, dolomía,
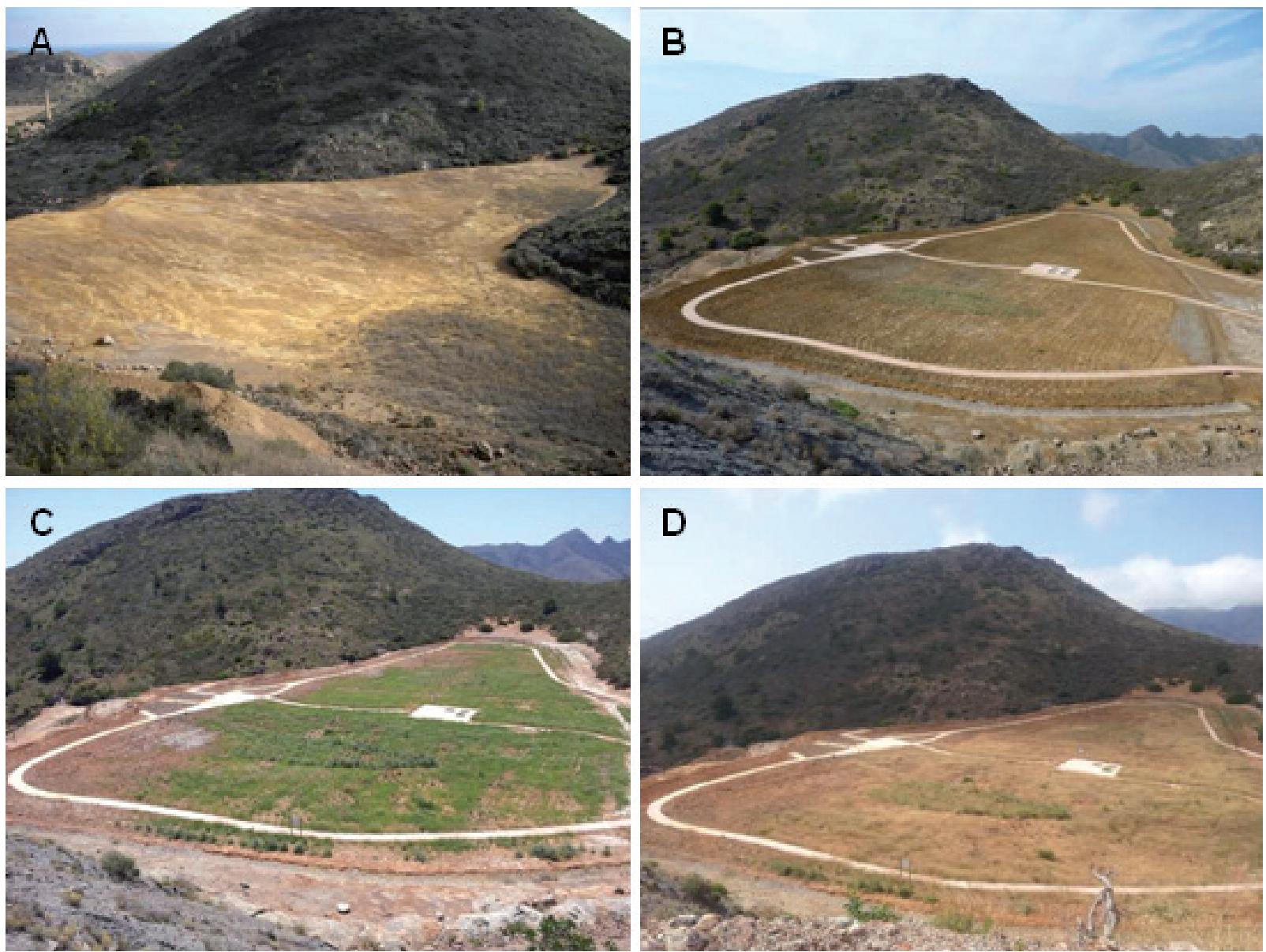

Figura 7. Imágenes de la superficie de la presa de residuos mineros Santa Antonieta antes de iniciar la estrategia de rehabilitación (octubre 2010) (A), 12 meses después de la primera adición de enmiendas (julio 2012) (B), 18 meses después de la primera adición de enmiendas (enero 2013) (C) y 24 meses después de la primera adición de enmiendas (julio 2013) (D).

Figure 7. Images of the surface of the Santa Antonieta tailings pond before the rehabilitation (October 2010) (A), 12 months after the first addition of amendments (July 2012) (B), 18 months after the first addition of amendments (January 2013) (C) and 24 months after the first addition of amendments (July 2013) (D). 
mármol), y de gran producción de residuos orgánicos como purines de cerdo, ya que los residuos generados pueden ser utilizados como enmienda para la rehabilitación de suelos afectados por metales pesados sin ningún coste salvo el relativo al transporte, transformando un residuo en un subproducto. La valorización de los residuos es esencial para el desarrollo sostenible, ya que el uso de vertederos debe minimizarse para evitar impactos ambientales negativos. Al mejorar la calidad del suelo con la aplicación de enmiendas, se ha conseguido una cubierta vegetal del $65 \%$, capaz de mantener a niveles bajos la fracción biodisponible de metales pesados. En la Figura 7 se puede apreciar las diferentes fases del proceso de rehabilitación en imágenes antes y después de las diferentes enmiendas aplicadas.

\section{Agradecimientos}

Este trabajo ha sido cofinanciado por el proyecto de la Unión Europea LIFE+ MIPOLARE (LIFE09 ENV/ES/000439).

\section{Referencias}

Alvarenga, P., Palma, P., Gonçalves, A.P., Baião, N., Fernandes, R.M., de Varennes, A., Vallini, G., Duarte, E. and Cunha-Queda, A.C. 2008. Assessment of chemical, biochemical and ecotoxicological aspects in a mine soil amended with sludge of either urban or industrial origin. Chemosphere, 72 (11), 1774-1781.

Baldock, J.A. and Skjemstad, J.O. 2000. Role of the soil matrix and minerals in protecting natural organic materials against biological attack. Organic Geochemistry, 31, 697-710.

Barker, A.V. 1997. Composition and uses of compost. En: Rechling J.E. (ed.), Agricultural uses of by-products and wastes. ACS Symposium Series No. 668, Vol 10, American Chemical Society, Washington DC, 140-162.

Bouwman, and Vangronsveld, J. 2004. Rehabilitation of the nematode fauna in a phytostabilized heavily zinc-contaminated, sandy soil. Journal of Soils and Sediments, 4 (1), 17-23.

Bradshaw, A.D. and Johnson, M. 1992. Revegetation of Metalliferous Mine Waste: The Range of Practical Techniques Used in Western Europe. Elsevier, Manchester.

Brallier, S., Harrison, R.B., Henry, C.L. and Dongsen, X. 1996. Liming effects on availability of $\mathrm{Cd}, \mathrm{Cu}, \mathrm{Ni}$ and $\mathrm{Zn}$ in soil amended with sewage sludge 16 years previously. Water, Air and Soil Pollution, 86, 195-206.

Brown, S.L., Sprenger, M., Maxemchuk, A. and Compton, H. 2005. Ecosystem function in alluvial tailings after biosolids and lime application. Journal of Environmental Quality, 34, 1-6.
Bukert, A., Bationo, A. and Possa, K. 2000. Mechanism of residue Mulch-induced cereal growth increases in West Africa. Soil Science Society of America Journal, 64, 1-42.

Chapman, H.D. 1965. Cation Exchange Capacity. En: Black C.A. (ed.), Methods of Soil Analysis. American Society of Agronomy, Madison, Wisconsin, 891-900.

Clough, A. and Skjemstad, J.O. 2000. Physical and chemical protection of soil organic carbon in three agricultural soils with different contents of calcium carbonate. Australian Journal of Soil Research, 38, 1005-1016

Conesa, H.M., Faz, A. and Arnaldos, R. 2006. Heavy metal accumulation and tolerance in plants from mine tailings of the semiarid Cartagena-La Union mining district (SE Spain). Science of the Total Environment, 366, 1-11.

Fernández-Caliani, J.C. and Barba-Brioso, C. 2010. Metal immobilization in hazardous contaminated miesoils after marble slurry waste application. A field assessment at the Tharsis mining disctrict (Spain). Journal of Hazardous Materials, 181 (1-3), 817-826.

Kabas, S., Faz, A., Acosta, J.A., Zornoza, R., MartínezMartínez, S., Carmona, D.M. and Bech, J. 2012. Effect of marble waste and pig slurry on the growth of native vegetation and heavy metal mobility in a mine tailing pond. Journal of Geochemical Exploration, 123, 69-76.

Kabas, S., Arocena, J.M., Acosta, J.A., Faz, A., MartínezMartínez, S., Zornoza, R. and Carmona, D.M. 2013. Syrian bean-caper (Zygophyllum fabago L.) improves organic matter and other properties of mine wastes deposits. International Journal of Phytoremediation, 16, 366-378.

Liu, L., Chen, H., Cai, P., Liang W. and Huang, O. 2009. Immobilization and phytotoxicity of $\mathrm{Cd}$ in contaminated soil amended with chicken manure compost. Journal of Hazardous Materials, 163 (2-3), 563-567.

Martínez, M.J. and Pérez, C. 2007. Niveles de fondo y niveles genéricos de referencia de metales pesados en suelos de la Región de Murcia. Comunidad Autónoma de la Región de Murcia, Murcia.

McLean, J. E. and Bledsoe, B.E. 1992. Behavior of Metals in Soils. Ground Water Issue. U.S. EPA. EPA/540/S-92/018.

Méndez, M.O. and Maier, R.M. 2008. Phytostabilization of mine tailings in arid and semiarid environments - an emerging remediation technology. Environmental Health Perspectives, 116 (3), 278-283.

Pardo, T., Clemente, R. and Bernal, M.P. 2011. Effects of compost, pig slurry and lime on trace element solubility and toxicity in two soils differently affected by mining activities. Chemosphere 84 (5), 642-650.

Pérez de Mora, A., Ortega-Calvo, J.J., Cabrera, F. and Madejón, E. 2005. Changes in enzyme activities and microbial biomass after "in situ" remediation of a heavy metal-contaminated soil. Applied Soil Ecology, 28 (2), 125-137.

Pérez de Mora, A., Burgos, P., Madejón, E., Cabrera, F., Jaeckel P. and Schloter, M. 2006. Microbial community structure and function in a soil contaminated by heavy metals: effects of plant growth and different amendments. Soil Biology \& Biochemistry, 38 (2), 327-341.

Pueyo, M., López-Sanchez, J.F. and Rauret, G. 2004. Assessment of $\mathrm{CaCl}_{2}, \mathrm{NaNO}_{3}$ and $\mathrm{NH}_{4} \mathrm{NO}_{3}$ extraction pro- 
cedures for the study of $\mathrm{Cd}, \mathrm{Cu}, \mathrm{Pb}$ and $\mathrm{Zn}$ extractability in contaminated soils. Analytica Chimica Acta, 504 (2), 217-226.

Risser, J.A. and Baker, D.E. 1990. Testing soils for toxic metals. En: Westerman, R. L. (ed.), Soil Testing and plant analysis. Soil Science Society of America Special Publication 3, Madison, Wisconsin, 275-298.

Shafi, M., Bakht, J., Jan, M.T. and Shah, Z. 2007. Soil C and $\mathrm{N}$ dynamics and maize (Zea mays L.) yield as affected by cropping systems and residue management in Northwestern Pakistan. Soil and Tillage Research, 94 (2), 520-529.

Simón, M., Martín, F., García, I., Bouza, P., Dorronsoro, C. and Aguilar, J. 2005. Interaction of limestone grains and acidic solutions from the oxidation of pyrite tailings. Environmental Pollution, 135 (1), 65-72.

Six, J., Bossuyt, H., Degryze, S. and Denef, K.2004. A history of research on the link between aggregates, soil biota, and soil organic matter dynamics. Soil \& Tillage Research, 79 (1), 7-31.

Smith, J.L., Papendick, R.I., Bezdicek, D.F. and Lynch, J.M. 1993. Soil organic matter dynamics and crop residue management. En: Meeting Jr, F.B. (ed.), Soil Microbial Ecology Application in Agricultural and Environmental Management. Marcel Dekker Inc., New York, 65-94.
Sobek, A.A., Schuller, W.A., Freeman, J.R. and Smith, R.M. 1978. Field and laboratory methods applicable to overburdens and mine soils. EPA-600/2-78-054.

USDA. 1999. Soil quality Test Kit Guide. United Stated Department of Agriculture, Washington.

Ye, Z.H., Shu, W.S., Zhang, Z.Q., Lan, C.Y. and Wong, M.H. 2002. Evaluation of major constraints to revegetation of lead/zinc mine tailings using bioassay techniques. Chemosphere, 47 (10), 1103-1111.

Zanuzzi, A., Arocena, J.M., van Mourik, J.M. and Faz, A. 2009. Amendments with organic and industrial wastes stimulate soil formation in mine tailings as revealed by micromorphology. Geoderma, 154 (1-2), 69-75.

Zornoza, R., Carmona, D.M., Acosta, J.A., MartínezMartínez, S. Weiss N. and Faz, A. 2011. The effect of former mining activities on contamination dynamics in sediments, surface water and vegetation in El Avenque stream, SE Spain. Water, Air and Soil Pollution, 223 (2), 519-532.

Zornoza, R., Faz, A., Carmona, D.M., Acosta, J.A., MartínezMartínez, S. and de Vreng, A. 2013. Carbon mineralization, microbial activity and metal dynamics in tailing ponds amended with pig slurry and marble waste. Chemosphere, 90 (10), 2606-2613.

Recibido: diciembre 2015

Revisado: febrero 2016

Aceptado: abril 2016

Publicado: junio 2017 
